\title{
ASPECTOS CLÍNICOS DA LEISHMANIOSE VISCERAL CANINA NA CIDADE DE FORTALEZA, CE
}

\author{
Clinical features of canine visceral leishmaniasis at Fortaleza city, Ceará, Brazil \\ CAMINHA, A.E.Q. ${ }^{1}$; SOTO-BLANCO, B. ${ }^{2}$ \\ ${ }^{1}$ Médico Veterinário, Fortaleza, CE \\ ${ }^{2}$ Departamento de Ciências Animais, Universidade Federal Rural do Semi-Árido -UFERSA, Mossoró, RN. \\ Endereço para correspondência: E-mail: bsotoblanco@yahoo.com.br. Fone: +55 84 3315-1760.
}

\section{RESUMO}

A leishmaniose visceral é uma antropozoonose muito importante no Brasil. O presente trabalho teve como objetivo realizar a descrição clínica de 21 cães naturalmente acometidos por leishmaniose visceral na cidade de Fortaleza, Ceará. O diagnóstico de leishmaniose visceral foi realizado por meio de exames sorológicos. As alterações mais freqüentes foram as dermatológicas, seguidas por emaciação, hiporexia, linfadenomegalia e apatia. Também foram observadas alterações oculares, esplenomegalia, artrite, onicogrifose, emese, miosite, desidratação, epistaxe e comprometimento do sistema urinário.

Palavras-chave: Leishmania chagasi, Leishmania infantum, cães, diagnóstico.

\section{ABSTRACT}

Visceral leishmaniasis is an infectious zoonosis with high importance in Brazil. The purpose of the present study was to evaluate the clinical findings of 21 dogs naturally infected by visceral leishmaniasis, in Fortaleza, Ceará, Brazil. The diagnosis was based on serology. Most of the dogs showed skin abnormalities, weight loss, decreased appetite, lymphadenomegaly and apathy. It was also observed ocular signs, splenomegaly, arthritis, onychogryphosis, emesis, myositis, dehydration, epistaxis, and involvement of the urinary system.

Key words: Leishmania chagasi, Leishmania infantum, dogs, diagnostics.

\section{INTRODUÇÃO}

A Leishmaniose Visceral, conhecida popularmente como calazar, necessita de um hospedeiro que aloje 0 agente infeccioso. Esse agente, Leishmania sp., é introduzido no organismo dos hospedeiros (homem ou animal) através de um vetor biológico infectado (insetos), que também servem de hospedeiros, onde o agente cumpre parte do seu ciclo de vida. Já foram descritas outras formas de transmissão excepcionais, tais como: direto de cão a cão, inter-humanos, picada de carrapatos, congênito e por transfusão sanguínea 
(Moreno e Alvar, 2002; Rosypal et al., 2003).

Nas Américas, o calazar é encontrado no México, El Salvador, Guatemala, Venezuela, Colômbia, Guiana Holandesa, Peru, Paraguai, Bolívia, Argentina e Brasil. O Brasil é responsável por mais de $90 \%$ dos casos relatados nas Américas (Alencar et al., 1991). A doença já foi descrita em 19 Estados do Brasil: Roraima, Paraná, Tocantins, Minas Gerais, Espírito Santo, Rio de Janeiro, São Paulo, Goiás, Mato Grosso, Mato Grosso do Sul e todos os Estados do Nordeste, sendo estes com a grande maioria dos casos registrados, e vem se apresentando em franca expansão pelo país (Castro, 1996; Oliveira e Santa Rosa, 1997).

O presente trabalho teve como objetivo realizar a descrição clínica de 21 cães da cidade de Fortaleza, Ceará, naturalmente acometidos por leishmaniose visceral, e verificar quais as alterações mais freqüentes, que possam auxiliar na identificação de casos da doença.

\section{MATERIAL E MÉTODOS}

Neste estudo foram utilizadas 21 fichas clínicas de cães naturalmente acometidos por leishmaniose visceral, oriundas de cinco diferentes clínicas. Estes prontuários foram extraídos de 170 fichas clínicas padronizados para a coleta dos dados exigidos encaminhadas a 15 clínicas veterinárias da cidade de Fortaleza, Ceará, no período de julho de 2003 a abril de 2004. Para inserção nesta pesquisa, os cães deveriam apresentar algum sinal clínico compatível com a doença e comprovação através de exames laboratoriais para o Calazar.

Em todos os animais, a confirmação do diagnostico de leishmaniose foi realizado por meio do resultado positivo de três exames sorológicos para cada animal: fixação de complemento (reativo 1/160), ELISA (positivo) e imunofluorescência indireta (reativo 1/160). Todos os exames foram realizados no Laboratório de Sorologia do Departamento de Parasitologia da Universidade Federal de Minas Gerais (UFMG).

As fichas clínicas analisadas foram de cães que apresentavam algum tipo de sinal clínico, isto é, eram oligossintomáticos ou polissintomáticos. Foram coletados os dados referentes a aspecto geral (temperatura, mucosas, presença de diarréia, emese, emaciação, hiporexia, ascite, edema, epistaxe, outros), achados à palpação (hepatomegalia, esplenomegalia, linfadenopatia, outras), afecções oftalmológicas (conjuntivite, blefarite, ceratite, ceratoconjuntivite seca, uveite, outras), distúrbios dermatológicos (alopecia, dermatite esfoliativa, ulceração, hiperqueratose nasal, hiperqueratose digital, nódulos, onicogrifose, outros; localização das lesões), alterações do aparelho locomotor (poliartrite, polimiosite, sinovite, outras), distúrbios renais (polidipsia, poliúria, oligúria, anúria, hematúria, outros), alterações neurológicas (encefalite, coroidite, outras) e outras observações. Dos 21 cães analisados, 14 animais foram avaliados através de eritrograma, leucograma e contagem de plaquetas.

\section{RESULTADOS E DISCUSSÃo}

Dos 21 cães avaliados $76 \%$ eram machos e $24 \%$ fêmeas. Não foi realizada uma avaliação etária, assim como a verificação do início dos sinais e a progressão dos mesmos, devido à falta de precisão no registro destes dados. As alterações clínicas dos 21 cães estão apresentadas na tabela 1. Em 20 destes animais foram observadas lesões dermatológicas, apresentadas na tabela 2 . Os achados laboratoriais dos 14 cães analisados estão presentes na tabela 3.

No presente estudo, foi encontrada uma grande disparidade no número de casos entre machos e fêmeas, o que não confirma os relatos da literatura (Rosypal et 
Tabela 1 - Alterações clínicas em 21 cães da cidade de Fortaleza-CE, naturalmente acometidos por leishmaniose visceral, entre julho de 2003 e abril de 2004.

\begin{tabular}{lc}
\hline \multicolumn{1}{c}{ Alteração clínica } & Freqüência \\
\hline Lesões dermatológicas & $20(95 \%)$ \\
Emaciação & $13(62 \%)$ \\
Hiporexia & $9(43 \%)$ \\
Linfadenopatia & $9(43 \%)$ \\
Apatia & $8(38 \%)$ \\
Esplenomegalia & $5(24 \%)$ \\
Artrite & $4(19 \%)$ \\
Onicogrifose & $3(14 \%)$ \\
Emese & $3(14 \%)$ \\
Secreção ocular aumentada & $3(14 \%)$ \\
Diarréia & $3(14 \%)$ \\
Miosite & $2(10 \%)$ \\
Desidratação & $2(10 \%)$ \\
Epistaxe & $2(10 \%)$ \\
Polidipsia e poliúria & $1(5 \%)$ \\
Blefarite & $2(10 \%)$ \\
Hepatomegalia & $2(10 \%)$ \\
Conjuntivite & $1(5 \%)$ \\
Edema & $1(5 \%)$ \\
Encefalite & $1(5 \%)$ \\
Otite bilateral & $1(5 \%)$ \\
\hline
\end{tabular}

Tabela 2 - Lesões dermatológicas em 20 cães da cidade de Fortaleza-CE, naturalmente acometidos por leishmaniose visceral, entre julho de 2003 e abril de 2004.

\begin{tabular}{lc}
\hline \multicolumn{1}{c}{ Lesão dérmica } & Freqüência \\
\hline Lesões ulcerativas & $6(30 \%)$ \\
Dermatite esfoliativa & $5(25 \%)$ \\
Hiperqueratose digital & $3(15 \%)$ \\
Alopecia & $2(10 \%)$ \\
Hiperqueratose nasal & $2(10 \%)$ \\
Pododermatite & $1(5 \%)$ \\
\hline
\end{tabular}

Tabela 3 - Achados laboratoriais em 14 cães da cidade de Fortaleza-CE, naturalmente acometidos por leishmaniose visceral, entre julho de 2003 e abril de 2004.

Hiperproteinemia

Trombocitopenia

Linfócitos reativos

Anemia normocítica-normocrômica

Rouleaux eritrocitário

Anemia normocítica-hipocrômica

Anisocitose

Leucocitose

Plaquetas gigantes

Linfopenia

Eosinopenia

Neutrofilia

Linfocitose

Hipoproteinemia
Freqüência

$10(71 \%)$

$10(71 \%)$

$10(71 \%)$

$8(57 \%)$

$7(50 \%)$

$5(36 \%)$

$4(29 \%)$

$4(29 \%)$

$3(21 \%)$

$3(21 \%)$

$2(14 \%)$

$1(7 \%)$

$1(7 \%)$

$1(7 \%)$ 
al., 2003; Feitosa et al., 2000). Por outro lado, a casuística aqui apresentada é muito pequena para confirmar essa diferença.

Como o objetivo deste relato foi a descrição clínica de cães com leishmaniose visceral, não foram incluídos animais assintomáticos. Portanto, o levantamento das fichas foi somente dos casos sintomáticos e que foram comprovados através de exames laboratoriais. Tal procedimento impossibilita que se verifique a porcentagem destes animais em relação aos soropositivos.

Os linfonodos mais comumente afetados foram os poplíteos $(89 \%$ dos casos com linfadenopatia), o que se compara com relato de Feitosa et al. (2000), apesar Nieto et al. (1992) terem evidenciado principalmente linfadenomegalia préescapular. Tal fato sugere evidências de envolvimento visceral, pelo fato de os poplíteos estarem em uma região mais próxima e problemas sistêmicos afetarem também esses gânglios, diferente dos préescapulares os quais são referidos a um maior número de lesões cutâneas na região cranial dos animais estudados (Nieto et al., 1992).

Com relação às alterações dermatológicas, os achados mais freqüentes foram lesões ulcerativas, acompanhadas de áreas de alopecia variável. $\mathrm{Na}$ maioria dos casos, estas lesões foram observadas em regiões de saliências ósseas $(70 \%$ dos casos com lesões dermatológicas), que se compara ao trabalho de Feitosa et al. (2000), que foi em torno de $50 \%$, e confirmando em parte outros trabalhos (Marzochi et al., 1985; Genargo, 1993) que constaram úlceras em diferentes regiões, mas preferencialmente nas saliências ósseas, plano nasal, bordas das pinas e espaços interdigitais. No plano nasal foram observados dois casos de hiperqueratose e no plano interdigital foram observados três casos de hiperqueratose e um caso de pododermatite.
Não foi verificada a ocorrência de prurido associada à presença de descamação cutânea, como relatada anteriormente (Feitosa et al., 2000). Apenas em alguns animais observou-se descamação cutânea localizada na região periocular (lesões em luneta) e nas bordas das pinas e na maioria dos casos, a descamação foi generalizada, como o observado por Greene e Slappendel (1993) e Genaro (1993).

A maior parte dos animais que apresentavam emaciação também manifestava atrofia da musculatura temporal. Existem relatos que registraram miotrofia da musculatura das fossas temporais em animais com leishmaniose visceral (Rosypal et al., 2003).

A anemia evidenciada pelas mucosas pálidas foi confirmada hematologicamente, sendo de caráter arregenerativo em quase todos os casos examinados. Sugere-se ter ocorrido por uma hipoplasia ou aplasia medular (Pocai et al., 1998). Dentre as alterações oculares, os casos de conjuntivite e blefarite foram menos aparentes, estando presente na maioria apenas secreção ocular, o que não se compara com observações feitas em Araçatuba, onde foi verificada maior incidência de uveíte, seguida de ceratoconjuntivite bilateral (Feitosa et al., 2000), e das obsevações de Viana Neto (2002) que, também em Fortaleza, verificaram uma incidência de conjuntivite $(70,9 \%)$, blefarite $(43,8 \%)$, ceratite $(35,3 \%)$, ceratoconjuntivite seca $(16,3 \%)$ e uveíte $(12,8 \%)$.

Os dois casos de epistaxe apresentavam um quadro de pancitopenia, sugerindo como causa um processo de hipoplasia ou aplasia medular, conforme sugerido por Pocai et al. (1998). Houve apenas um caso de leishmaniose visceral que desenvolveu encefalite, apresentando alterações neurológicas caracterizadas por deambulação para o lado esquerdo, pupilas com reflexo reduzido e midriase e miose alternados em cada pupila. Ocorreram 
ainda alterações osteo-musculares, sendo quatro casos de artrite e dois casos de miosite.

\section{CONCLUSÕES}

Há uma grande variedade de sinais clínicos associados à leishmaniose visceral, destacando alterações dermatológicas, emaciação, hiporexia, linfadenomegalia e apatia. Também foram observadas alterações oculares, esplenomegalia, artrite, onicogrifose, emese, miosite, desidratação, epistaxe e comprometimento do sistema urinário.

\section{REFERÊNCIAS}

ALENCAR, J. E.; DIETZE, R.; NEVES, J. Leishmaniose visceral (calazar). In: VERNESI, R.; FOCACCIA, R.; DIETZE, R. Doenças Infecciosas e Parasitárias, Rio de Janeiro: Guanabara-Koogan, 1991. p. 706-716.

CASTRO, A. G. Controle, Diagnóstico e Tratamento da Leishmaniose Visceral (Calazar) Normas Técnicas, Brasília: Fundação Nacional de Saúde, 1996. 86p.

FEITOSA, M. M.; IKEDA, F. A.; LUVIZOTTO, M. C. R.; PERRI, S. H. V. Aspectos clínicos de cães com leishmaniose visceral no município de Araçatuba São Paulo (Brasil). Clínica Veterinária, n.28, p.3644, 2000.

GENARO, O. Leishmaniose Visceral Canina Experimental. 1993. 202 p. Tese (Doutorado) Faculdade de Medicina Veterinária e Zootecnia, Universidade Federal de Minas Gerais, Belo Horizonte, 1993.

GREENE, C. E.; SLAPPENDEL, R. J. Leishmaniasis. In: GREENE, C. E. Clinical
Microbiology and Infectious Diseases of the Dog and Cat, Philadelphia: W. B. SAUNDERS Co., 1990. p. $450-8$.

MARZOCHI, M. C.; COUTINHO, S. G.; SOUZA, W. J.; TOLEDO, L. M.; GRIMALDI JÚNIOR, G.; MOMEN, H.; PACHECO, R. S.; SABROZA, P. C.; SOUZA, M. A.; RANGEL, F. B. F.; TRAMONTANO, N. C. Canine visceral leishmaniosis in Rio de Janeiro, Brazil. Clinical, parasitological, therapeutical and epidemiological findings (19771983). Memórias do Instituto Oswaldo Cruz, v.80, n.3, p.349-357, 1985.

MORENO, J.; ALVAR, J. Canine leishmaniasis: epidemiological risk and the experimental model. Trends in Parasitology, v.18, n.9, p.399-405, 2002.

NIETO, C. G.; NAVARRETE, I.; HABELA, M. A.; SERRANO, F.; REDONDO, E. Pathological changes in kidneys of dogs with natural Leishmania infection. Veterinary Parasitology, v. 45, n. 1-2, p. 33-47, 1992.

OLIVEIRA, I. C. S.; SANTA ROSA, I. C. A. Leishmaniose visceral: breve revisão sobre uma zoonose reemergente. Clínica Veterinária, n. 11, p. 24-28, 1997.

POCAI, E. A.; FROZZA, L.; HEADLEY, S. A.; GRAÇA, D. L. Leishmaniose visceral (calazar): cinco casos em cães de Santa Maria, Rio Grande do Sul, Brasil. Ciência Rural, v. 28 , n. 3, p. 501505, 1998.

ROSYPAL, A. C.; ZAJAC, A. M.; LINDSAY, D. S. Canine visceral leishmaniasis and its emergence in the United States. Veterinary Clinics of North America, Small Animals Practice, v.33, n.4, p.921937, 2003.

VIANA NETO, R. L. Bases epidemiológicas da leishmaniose canina na cidade de Fortaleza Ce. 2002. 41p. Trabalho de conclusão de curso (Especialização em saúde pública) - Centro de Ciências da Saúde, Universidade Estadual do Ceará, Fortaleza, 2002. 